\title{
Seminare 2004
}

\section{Praxisübergabe/-übernahme}

\section{Teilnehmende}

Das Seminar richtet sich an zukünftige Praxisübergeber/innen sowie Praxisübernehmer/innen. Hinweis: Für Praxisübergeber/innen lohnt es sich, sich bereits frühzeitig (5-10 Jahre) mit diesem Thema auseinanderzusetzen.

\section{Themen}

Juristische Aspekte (Übergabe der Krankengeschichten, Abschluss Praxisübernahmevertrag, Mietvertrag), Ehe- und Erbrecht (Ehegüterstand, Erbschaftsfolge/-planung), Versicherungen (Übergabe/Übernahme und Abschluss/Auflösung von Versicherungsverträgen, Vorsorgeplanung), Bewertung einer Arztpraxis (Berechnung Inventarwert und Goodwill als Verhandlungsbasis), Steuern (Liquidations- und Grundstückgewinnsteuer, Steuerfolgen bei Aufnahme der Selbständigerwerbstätigkeit, Bestimmung des optimalen Übergabezeitpunkts).

\section{Unterlagen}

Die Seminarteilnehmer/innen erhalten einen Seminarordner mit Unterlagen zu den behandelten Themen.

\section{Kosten / Sponsoren}

Das Seminar ist für FMH-Mitglieder gratis. Die Kosten werden von folgenden Sponsoren gedeckt: Versicherung der Schweizer Ärzte, Schweizerische Ärzte-Krankenkasse und AstraZeneca AG.

\section{Daten}

\begin{tabular}{llll} 
Nr. 06 & \multicolumn{2}{c}{$\begin{array}{l}\text { Donnerstag, 17. Juni } 2004 \\
\text { Bern }\end{array}$} & 9.30-16.30 Uhr \\
\hline Nr. 07 & $\begin{array}{l}\text { Donnerstag, 9. September 2004 Bern } \\
\text { Zürich }\end{array}$ & FMT Zürich \\
\hline
\end{tabular}

\section{Anmeldung}

Sie können sich bei nachfolgend aufgeführter Adresse (FMH Consulting Services) oder via Internet unter www.fmhservices.ch einschreiben.

\section{Buchhaltung und Steuern}

\section{Teilnehmende}

Das Seminar richtet sich an Praxiseröffner/innen, Praxisübernehmer/innen sowie an bereits praxistätige Ärztinnen und Ärzte.

\section{Themen}

Im Buchhaltungsteil erfahren die Seminarteilnehmer/innen, welche Massnahmen vor der Praxiseröffnung getroffen werden müssen und welches deren organisatorischen Voraussetzungen sind. Basierend auf dem Standardkontenplan für Ärzte wird die Führung einer doppelten Buchhaltung aufgezeigt. Zudem erhalten sie Einblick in eine EDV-unterstützte Buchführungslösung. Im Steuerteil werden die Methoden der Steuerplanung aus versicherungstechnischer sowie aus treuhänderischer Sicht aufgezeigt.

\section{Unterlagen}

Die Seminarteilnehmer/innen erhalten einen Seminarordner mit Unterlagen zu den behandelten Themen.

\section{Kosten}

Das Seminar ist für FMH-Mitglieder gratis.

\section{Daten}

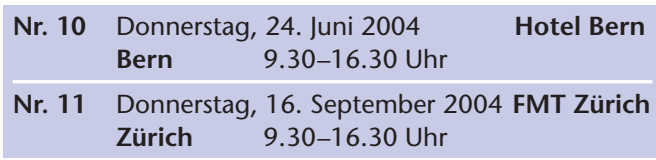

\section{Anmeldung}

Sie können sich bei nachfolgend aufgeführter Adresse (FMH Consulting Services) oder via Internet unter www.fmhservices.ch einschreiben.

\section{Praxiscomputer-Workshop}

\section{Teilnehmende}

Der Workshop richtet sich an praxiseröffnende sowie an bereits praxistätige Ärztinnen und Ärzte.

\section{Inhalt}

Die Seminarteilnehmer/innen erhalten die Gelegenheit, die Praxisadministrationssoftware von sechs EDV-Unternehmen kennenzulernen. Sie erhalten einen Einblick in TARMED-kompatible Lösungen für die Leistungsabrechnung, die Führung elektronischer Krankengeschichten, die Einbindung von Laborgeräten und von Finanzbuchhaltungslösungen. Bei den anwesenden Firmen handelt es sich jeweils um fünf Hersteller von EDV-Gesamtlösungen in der Arztpraxis sowie um eine Abrechnungskasse. 


\section{Ziel}

Die Workshopteilnehmer/innen erhalten einen ersten Überblick über führende Softwarelösungen. Sie erfahren, wie bei der Evaluation eines Ärztepaketes zweckmässigerweise vorgegangen wird und welche Fehler vermieden werden sollten.

\section{Kosten}

Der Workshop ist für FMH-Mitglieder gratis.

\section{Daten}

$\begin{array}{lll}\text { Nr. } 14 & \text { Donnerstag, 1. Juli } 2004 \\ \text { Bern } & 14.00-17.30 ~ U h r\end{array}$

\section{Folgende Softwareanbieter können Sie kennenlernen:}

\section{Ärztekasse, Urdorf ZH (MediWin CB Pro)}

Als Marktleader seit 1964 im Bereich Praxisadministration können Sie bei der Ärztekasse auf eine ganzheitliche Lösung für Ihre Fragen betreffend Abrechnungsvarianten, Computerwahl, Netzwerke, Formular- und Briefbearbeitung etc. zählen. Die statistischen Erhebungen (im Auftrag der FMH, Roko) sind ein anerkanntes betriebswirtschaftliches und standesorganisatorisches Hilfsmittel für die moderne Praxisführung. Nebst innovativen Ideen und Lösungen sowie Kooperationspartnerschaft mit 10 TrustCentern ist uns eine umfassende Kundenbetreuung ein Anliegen. Unsere Standorte befinden sich in Basel, Bern, Chur, Genf, Luzern, Le Landeron, Lugano, St. Gallen und Zürich. Sie definieren Ihre Wünsche - wir bieten Ihnen die Lösung!

\section{Delemed AG, Gümligen (delemed ${ }^{\circledR}$ PEX II)}

Delemed AG entwickelt und vertreibt seit 13 Jahren erfolgreich PEX II, die einfache, sympathische und effiziente Software für die Arztpraxis und Kliniken. Durch einen modularen Aufbau kann das System jederzeit den aktuellen Bedürfnissen des Kunden angepasst werden. Dank unserer Vielseitigkeit im medizinischen Umfeld und unseres Kundenstamms von über 590 Anwendern sind wir ein optimaler Partner für Sie.

\section{Kern Concept, Gossau (Aeskulap)}

Aeskulap ist ein extrem schnelles Windows-Programm, das zusammen mit einer SQL-Datenbank höchste Verarbeitungsgeschwindigkeit, Kompaktheit und schnellste Zugriffsmöglichkeiten gewährleistet. Aeskulap bietet für alle Bedürfnisse eine optimale Lösung: vom einfachen Abrechnungssystem mit sämtlichen Leistungs- erfassungsmöglichkeiten bis zur vollelektronischen, papierlosen KG-Führung. Aeskulap ist ein modular aufgebautes, anpassbares System mit einer Vielzahl von Modulen. Als einziges Softwarehaus haben wir für den TARMED einen TARMED-Validator sowie einen TARMED-Optimizer direkt in die Ärztesoftware integriert. Die sofortige Validierung und Optimierung nach jeder Positionseingabe gewährleistet vollständige Rechnungen, da technische Grundleistungen, Prozentzuschläge, Alterszuschläge vollautomatisch hinzugefügt werden, ohne dass dafür dutzendfache Leistungsblöcke notwendig sind.

\section{Praxidata AG, Thun-Allmendingen (PRAXIDATA PraxiCenter ${ }^{\circledR}$ )}

Praxidata AG ist eines der führenden Systemhäuser für Medizininformatik und gehört seit Frühjahr 2002 zur Triamun-Gruppe. Praxidata steht für Sicherheit, Innovation und gute Lösungen, womit ein reibungsloser Ablauf im Praxisalltag gewährleistet ist. Mit den Praxidata-Modulen wurde eine wirtschaftliche Komplettlösung geschaffen, die sich den unterschiedlichen Bedürfnissen in den Arztpraxen optimal anpasst. Im Hinblick auf TARMED hat Praxidata mit der Neuentwicklung der Produktepalette PraxiCenter $^{\circledR}$ auf .NET-Technologie von Microsoft für ihre Kunden wiederum eine gute Lösung gefunden. Damit setzt das Unternehmen der TriamunGruppe jenseits herkömmlicher 32bit-Lösungen neue Massstäbe und sichert dadurch die Zukunft seiner Kunden.

\section{mathiesen \& mathiesen, Basel (NOVAX)}

Beurteilung vom Kunden: - «NOVAX ist ein umfassendes Werkzeug, unverzichtbar für jede Praxis!» (Dr. J. Lang); - «In unserer lebendigen Doppelpraxis ist die elektronische Agenda von NOVAX ein sehr wertvolles Instrument geworden und nicht mehr wegzudenken!» (Dr. H. J. Bumbacher); - «KG zum Abliefern nach Vertretung erledige ich jetzt dank NOVAX per Knopfdruck» (Dr. M. Kraszewski); - «Dank der Dokumentenverwaltung in NOVAX suche ich nie mehr Befunde und Berichte.» (Dr. B. Häfliger); «Mit einem Blick Übersicht über interne und externe medizinische Abklärungsschritte und wertvolle Gedankenstütze bei medizinischen differentialdiagnostischen Überlegungen.» (Dr. P. Lohrer); - «Das Laborfenster in NOVAX gefällt mir besonders gut; mit einem Klick habe ich die gesamten Labordaten meines Patienten auf dem Bildschirm!» (Dr. A. Häner); - «Für die Agenda in NOVAX gilt: Klicken ist schneller als blättern!» (Frau S. Grob) 
VarioSoft D. Cohen, Bern (mFmed EVOLUTION)

Variosoft konzentriert sich ausschliesslich auf den Ärztebereich. Benutzerfreundlichkeit, Effizienz, Ergonomie und Flexibilität sind nur einige der Stärken unserer echten 32-Bit-Applikation mFmed EVOLUTION.

\section{Anmeldung}

Sie können sich bei nachfolgend aufgeführter Adresse (FMH Consulting Services) oder via Internet unter www.fmhservices.ch einschreiben.

\section{Anmeldung und Auskunft}

FMH Consulting Services, Simone Köpfli, Burghöhe 1, 6208 Oberkirch, Tel. 04192500 77, Fax 0419210586 oder via Internet unter www.fmhservices.ch.

\section{Annullierungsbedingungen}

Bei den Gratisseminaren (exkl. PraxiscomputerWorkshop) werden bei Abmeldungen oder Fernbleiben folgende Unkostenbeiträge erhoben:

- Fr. 50.- pro Person ab 14 Tage vor Seminarbeginn;

- Fr. 80.- pro Person ab 7 Tage vor Seminarbeginn oder Fernbleiben. 